\title{
Gestaltungsdimensionen der Kommunikation von automatisierten Fahrzeugen und anderen Verkehrsteilnehmenden
}

\author{
Philip Joisten $^{1}$ - Anton Freund ${ }^{1}$ - Bettina Abendroth ${ }^{1}$ \\ Online publiziert: 16 . April 2020 \\ (c) Der/die Autor(en) 2020
}

\section{Zusammenfassung}

Bereits heute wird die Kommunikation zwischen automatisierten Fahrzeugen und anderen Straßenverkehrsteilnehmenden entwickelt und erforscht, um zukünftig einen sicheren und effizienten Straßenverkehrsfluss zu gewährleisten. Dieser Beitrag zielt darauf ab, Gestaltungsdimensionen der Kommunikation zwischen automatisierten Fahrzeugen und anderen Straßenverkehrsteilnehmenden herzuleiten und zu analysieren. Folgende Fragestellung steht im Vordergrund: Wie kann eine Kommunikationsstrategie zwischen automatisierten Fahrzeugen und anderen Straßenverkehrsteilnehmenden gestaltet werden? Zur Beantwortung dieser Fragestellung werden Gestaltungsdimensionen theoretisch abgeleitet und eine Befragung $(N=30)$ und Beobachtung $(N=308)$ von Fußgänger/-innen in Testfeldern von automatisierten Bussen durchgeführt. Aufbauend auf Erkenntnissen der Kommunikationstheorie werden sechs Gestaltungsdimensionen (Wer, Warum, In welcher Situation, Was, Wann, Wie) und dahinterstehende zentrale Fragestellungen identifiziert. Die Ergebnisse der empirischen Studien zeigen Verhaltensweisen und den Kommunikationsbedarf von Fußgänger/-innen bei der Interaktion mit automatisierten Bussen. Die Analyse anhand der Gestaltungsdimensionen legt weiteren Forschungsbedarf offen, wie z. B. nach den zukünftigen Rollen aller Beteiligten im Straßenverkehr.

Praktische Relevanz Die Anwendung des Frameworks von Gestaltungsdimensionen unterstützt bei der Gestaltung der Kommunikation von automatisierten Fahrzeugen, um zukünftig die notwendige Kooperation zwischen Straßenverkehrsteilnehmenden sicherzustellen.

Schlüsselwörter Kommunikation · Interaktion · Automatisierte Fahrzeuge · Fußgänger/-innen · Fahrzeug-Fußgänger-Kommunikation

Philip Joisten, M.Sc.

p.joisten@iad.tu-darmstadt.de

1 Fachbereich Maschinenbau, Institut für Arbeitswissenschaft, Technische Universität Darmstadt, Otto-Berndt-Straße 2, 64287 Darmstadt, Deutschland 


\title{
Design dimensions of the communication between automated vehicles and other road users
}

\begin{abstract}
Communication between automated vehicles and other road users is already being developed and researched today to ensure safe and efficient road traffic flow in the future. This paper aims to derive and analyse design dimensions of the communication between automated vehicles and other road users. The focus is on the following research question: How can a communication strategy between automated vehicles and other road users be designed? To answer this question, a framework of design dimensions is derived theoretically and a survey $(N=30)$ and observation $(N=308)$ of pedestrians in test fields of automated buses is carried out. Based on the findings of communication theory, six design dimensions (who, why, in which situation, what, when, how) and central questions behind them are identified. The results of the empirical studies show the behaviour and communication needs of pedestrians when interacting with automated buses. The analysis based on the design dimensions reveals a need for further research, e.g. into the future roles of all road users.

Practical Relevance The application of the framework supports the design of the communication of automated vehicles to ensure the necessary cooperation between road users in the future.
\end{abstract}

Keywords Communication · Interaction · Automated vehicles $\cdot$ Pedestrians $\cdot$ Vehicle-pedestrian-communication

\section{Hintergrund und Problemstellung}

Die Teilnahme am Straßenverkehr ist für viele Menschen zu Fuß, mit dem Fahrrad oder mit Fahrzeugen alltäglich. StraBenverkehrsteilnehmende kommunizieren untereinander mittels impliziter und expliziter Signale, wie beispielsweise durch die Regulierung der Geschwindigkeit und die Wahl der Trajektorie, sowie durch Blicke, Handgesten und die Betätigung des Fahrtrichtungsanzeigers (Dey und Terken 2017; Stanciu et al. 2018). Der Einsatz von Kommunikationssignalen dient der Kooperation zwischen Straßenverkehrsteilnehmenden, die insbesondere dann an Relevanz gewinnt, wenn Störungen im Straßenverkehr auftreten (Merten 1977). Störungen im Straßenverkehr entstehen beispielsweise in sogenannten Deadlock-Situationen, die eine gegenseitige Blockade von Straßenverkehrsteilnehmenden beschreiben (Imbsweiler et al. 2018a). Kooperation und Kommunikation im Straßenverkehr löst und beugt Störungen vor, trägt zur Sicherheit von Straßenverkehrsteilnehmenden bei und fördert einen effektiven und effizienten Straßenverkehrsfluss (Nuñes Velasco et al. 2019; Penmetsa et al. 2019).

Das Verhalten einzelner Straßenverkehrsteilnehmender wird bestimmt durch und ist abhängig vom Kollektiv aller Teilnehmenden am Straßenverkehr (Wilde 1976). Der Straßenverkehr ist ein sozialer Raum, in dem Menschen in anonymisierter Form miteinander kooperieren, interagieren und kommunizieren. Der Faktor Mensch ist im Straßenverkehr umgeben von Technologien, wie beispielsweise Fahrzeugen oder Lichtsignalanlagen. Dieser sozio-technische Raum ist eingebettet in eine Organisation, die sich über Rollen, Erwartungen und Routinen der Straßenverkehrsteilnehmenden und übergeordnete Regeln und Gesetze wiederspiegelt.
In Zukunft sollen automatisierte Fahrzeuge in den StraBenverkehr integriert werden (ERTRAC 2019). Diese Entwicklung verspricht den Nutzen einer höheren Effizienz, von mehr Sicherheit und der Inklusion mobilitätseingeschränkter Menschen (Fagnant und Kockelman 2015; Colley et al. 2019). Die Einführung automatisierter Fahrzeuge hat Einfluss auf den sozialen Raum, die eingesetzten Technologien und die Organisation des Straßenverkehrs. Dies betrifft auch die Kommunikation im Straßenverkehr, die sich traditionell zwischen Straßenverkehrsteilnehmenden, wie zu Fuß Gehende, Fahrrad Fahrende und Fahrende von Fahrzeugen, vollzieht. Der Wandel des Fahrenden eines Fahrzeugs zum Mitfahrenden eines automatisierten Fahrzeugs bedingt, dass die kommunikative Funktion des Fahrenden durch das technische System des automatisierten Fahrzeugs substituiert wird. Ein Unterlassen von Kommunikation durch das automatisierte Fahrzeug, zum Beispiel beim Auftreten von Störungen im Straßenverkehr, würde der mit der Automation angestrebten Erhöhung von Sicherheit und Effizienz im Straßenverkehr entgegenstehen.

Automatisierte Fahrzeuge besitzen heute noch nicht dieselben Fähigkeiten zur Kommunikation mit anderen StraBenverkehrsteilnehmenden, wie sie der Mensch durch die Verwendung und Interpretation von impliziten und expliziten Kommunikationssignalen hat (Stanciu et al. 2018). Damit Sicherheit und Akzeptanz sowie Vertrauen von anderen Straßenverkehrsteilnehmenden in automatisierte Fahrzeuge sichergestellt sind, müssen Kommunikationsstrategien zwischen automatisierten Fahrzeugen und anderen Straßenverkehrsteilnehmenden erforscht werden (Habibovic et al. 2018). Aktuelle Forschungen untersuchen, wie die Kommunikation zwischen automatisierten Fahrzeugen und anderen Verkehrsteilnehmenden durch technische Hilfsmittel ergänzt werden kann (vgl. Rasouli und Tsotsos 2020). 
Tab. 1 Gestaltungsdimensionen einer Kommunikationsstrategie zwischen Straßenverkehrsteilnehmenden

Table 1 Design dimensions of a communication strategy between road users

\begin{tabular}{|c|c|c|}
\hline Elemente nach Clark (2006) & Gestaltungsdimension & Zentrale Fragestellung (Erläuterung) \\
\hline Wer kommuniziert? & Wer & Wer sind die Beteiligten der Kommunikation? \\
\hline \multirow[t]{2}{*}{ Welche Rollen haben die Beteiligten inne? } & Warum & Welches Ziel hat die Kommunikation? \\
\hline & In welcher Situation & In welcher Situation wird kommuniziert? \\
\hline $\begin{array}{l}\text { Welche Handlungen sollen ausgeführt } \\
\text { werden? }\end{array}$ & Was & Was sind die Inhalte der Kommunikation? \\
\hline \multirow{2}{*}{$\begin{array}{l}\text { Wann und wo sollen diese Handlungen } \\
\text { ausgeführt werden? }\end{array}$} & Wann & Zu welchem Zeitpunkt wird kommuniziert? \\
\hline & Wie & $\begin{array}{l}\text { Auf welche Art und Weise werden die Inhalte der Kommunikation } \\
\text { übertragen? }\end{array}$ \\
\hline
\end{tabular}

In diesem Beitrag leiten wir Gestaltungsdimensionen der Kommunikationsstrategie zwischen automatisierten Fahrzeugen und anderen Verkehrsteilnehmenden her. Des Weiteren geben wir Ergebnisse einer Befragung und einer Beobachtung von Fußgänger/innen im Testfeld eines automatisierten Buses wieder. Der Beitrag verfolgt dabei zwei Ziele: Erstens wird ein Framework präsentiert, das die Gestaltung der Kommunikation zwischen automatisierten Fahrzeugen und anderen Straßenverkehrsteilnehmenden strukturiert. Neben der Betrachtung des gesamten sozialen Raums des Straßenverkehrs soll das Framework dazu dienen, einzelne Fragestellungen zu gliedern. Zweitens wird die Anwendung des Frameworks anhand empirischer Studien diskutiert, deren Ziel es ist, erste Einblicke in das Verhalten von Fußgänger/-innen bei der Interaktion mit automatisierten Fahrzeugen zu erhalten.

Der Beitrag ist wie folgt aufgebaut: In Abschn. 2 werden Gestaltungsdimensionen der Kommunikationsstrategie zwischen automatisierten Fahrzeugen und anderen Straßenverkehrsteilnehmenden aufgezeigt und der Stand der Forschung dargelegt. Abschn. 3 beschreibt die Methodik und Ergebnisse der empirischen Studien. Abschn. 4 und 5 beinhalten die Diskussion des Frameworks und der empirischen Studien und das Fazit des Beitrags.

\section{Die Kommunikationsstrategie zwischen automatisierten Fahrzeugen und anderen Verkehrsteilnehmenden}

Der Begriff Strategie umschreibt in der Spieltheorie einen vollständigen Plan darüber, wie sich eine spielende Person in jeder Spielsituation verhalten wird (Morgenstern 1968). In Anlehnung an diese Definition soll im Kontext des StraBenverkehrs der Begriff Kommunikationsstrategie den Plan umschreiben, nach dem Straßenverkehrsteilnehmende miteinander kommunizieren. Im Anwendungsfall des automatisierten Fahrens setzen sich die Kommunikationsbeteiligten aus einem automatisierten Fahrzeug (z.B. Personenkraftwagen (Pkw) oder Autobus (Bus)) und mindestens einem weiteren konventionellen Straßenverkehrsteilnehmen- den, wie beispielsweise Fußgänger/-innen, Fahrrad Fahrende oder Fahrende konventioneller Fahrzeuge, zusammen.

Kommunikation dient primär dazu, Kooperationsprobleme zu lösen und ein gemeinsames Vorgehen zu koordinieren (Bangerter und Mayor 2013). Dazu müssen die Beteiligten der Kommunikation nach Clark (2006, S. 135) fünf Elemente adressieren:

- Wer kommuniziert?

- Welche Rollen haben die Beteiligten inne?

- Welche Handlungen sollen ausgeführt werden?

- Wann und wo sollen diese Handlungen ausgeführt werden?

Das Erreichen einer Einigung über diese Elemente ist inkrementell und hierarchisch (Clark 2006). In diesem Sinne vollzieht sich Kommunikation schrittweise durch die Beantwortung der hinter den Elementen stehenden Fragestellungen. Dies führt zur Entstehung von kooperativen Aktivitäten.

Die aus der Kommunikationstheorie gewonnenen Erkenntnisse können auf den Kontext der Kommunikation zwischen automatisierten Fahrzeugen und anderen StraBenverkehrsteilnehmenden übertragen werden. Damit Kooperationsprobleme im Straßenverkehr von einem automatisierten Fahrzeug gelöst werden können, müssen die von Clark (2006) beschriebenen Elemente beachtet werden. Daraus können die in Tab. 1 aufgeführten Dimensionen der Gestaltung eines technischen Systems, das mit anderen Straßenverkehrsteilnehmenden kommunizieren soll, abgeleitet werden. Tab. 1 fasst diese Gestaltungsdimensionen und die zugrundeliegenden zentralen Fragestellungen zusammen. In den folgenden Abschnitten wird auf die einzelnen Gestaltungsdimensionen eingegangen.

\subsection{Wer kommuniziert?}

Zur Koordination von Handlungen zwischen Straßenverkehrsteilnehmenden sind die Beteiligten der Kommunikation eindeutig zu identifizieren. Wer im Straßenverkehr kommuniziert bestimmt sich oftmals durch die räumliche Distanz bzw. Anordnung von Straßenverkehrsteilnehmenden. 
Eine Störung des Verkehrsflusses durch eine gegenseitige Blockade an einer Kreuzung entsteht beispielsweise erst durch das gleichzeitige Aufeinandertreffen von mehreren Straßenverkehrsteilnehmenden an der Kreuzung. Hieraus leiten sich direkt die Fragen nach der Anzahl der Kommunikationsbeteiligten und der Kommunikationsrichtung ab.

Die Beantwortung der Frage nach den Beteiligten der Kommunikation ist das erste Element der Herausbildung von kooperativen Aktivitäten nach Clark (2006) und stellt zentrale Anforderungen an die Gestaltung der Kommunikation zwischen automatisierten Fahrzeugen und anderen Straßenverkehrsteilnehmenden. Aus der Perspektive eines automatisierten Fahrzeugs resultiert, dass verschiedene Straßenverkehrsteilnehmende identifiziert und unterschieden werden sollten. Erst durch eine gerichtete, eindeutig adressierte Kommunikation des automatisierten Fahrzeugs (vgl. Ackermann et al. 2019) kann die Frage nach den Beteiligten der Kommunikation für andere Straßenverkehrsteilnehmende hinreichend beantwortet werden. Des Weiteren muss aus der Perspektive von anderen Straßenverkehrsteilnehmenden eindeutig sichergestellt sein, ob das Fahrzeug selbst oder Mitfahrende des Fahrzeugs für die Kommunikation verantwortlich sind. Dies gewinnt insbesondere beim hochautomatisierten Fahren an Relevanz, weil Transitionen zwischen automatisierten und nicht-automatisierten Fahrmodus möglich sind. Eine mögliche Gestaltungslösung ist hierbei die Kommunikation des Automationsmodus gegenüber anderen Straßenverkehrsteilnehmenden (vgl. Joisten et al. 2019).

\subsection{Welche Rolle haben die Akteure inne?}

Nachdem die Beteiligten der Kommunikation bestimmt sind, determinieren die Rollen der Straßenverkehrsteilnehmenden die Herausbildung von kooperativen Handlungen. Mit der Rolle eines Straßenverkehrsteilnehmenden sind Ziele und Erwartungen verbunden, die bestimmen, wie sich Straßenverkehrsteilnehmende in einer Situation verhalten wollen (vgl. Imbsweiler et al. 2018b). Die Rollen der Straßenverkehrsteilnehmenden können aus bestimmten Funktionen, wie beispielsweise der Funktion eines Einsatzfahrzeugs mit Blaulicht oder eines Abfallsammelfahrzeugs, resultieren oder sie resultieren aus der bestehenden Organisation des Straßenverkehrs. Bestimmend für die Organisation des Straßenverkehrs sind formelle und informelle Regeln (vgl. Imbsweiler et al. 2018a), die die Kooperation zwischen Straßenverkehrsteilnehmenden prägen. Diese Regeln sind situationsabhängig und -gebunden, weshalb die Rollen von Straßenverkehrsteilnehmenden wechselnd und flexibel sind und sich aus der zugrundeliegenden Situation ergeben. Die situationsabhängige Regelung des Vorrangs und die damit einhergehende Rolle von Straßenverkehrsteilnehmenden bestimmt, ob und in welchem Ausmaß kooperative Handlungen zwischen Straßenverkehrsteilnehmenden benötigt werden.

Dem zweiten Element von Clark (2006) zur Herausbildung kooperativer Handlungen werden die Gestaltungsdimensionen „Warum“ und „In welcher Situation“ zugeordnet. Zur Gestaltung von Kommunikation zwischen automatisierten Fahrzeugen und anderen Straßenverkehrsteilnehmenden sollten Ziele und Erwartungen von Straßenverkehrsteilnehmenden berücksichtigt werden, die eng verbunden sind mit den informellen und formellen Regeln der zugrundeliegenden Situation im Straßenverkehr.

\subsection{Welche Handlungen sollen ausgeführt werden?}

Neben der Festlegung von Akteuren und Rollen (,Wer“, „Warum" und „In welcher Situation") ist ein Inhalt der Kommunikation (,Was") notwendig, um eine Einigung über eine kooperative Handlung zwischen Straßenverkehrsteilnehmenden zu erzielen. Der Kommunikationsinhalt ergibt sich aus dem Ziel der Kommunikation und kann sich auf bestimmte, geplante Manöver bzw. Bewegungen der StraBenverkehrsteilnehmenden beziehen, wie beispielsweise die Durchfahrt einer Engstelle als vorrangiges Fahrzeug. Ebenso kann der Kommunikationsinhalt in der Aufforderung zum Unterlassen einer bestimmten Handlung bestehen. Ein Pkw-Fahrer kann beispielsweise durch das Beibehalten seiner Geschwindigkeit einen Fußgänger oder einer Fußgängerin zum Unterlassen einer Straßenüberquerung auffordern.

Die Inhalte der Kommunikation werden durch die eingenommenen Rollen der Straßenverkehrsteilnehmenden bestimmt. Für die Gestaltung der Kommunikation zwischen automatisierten Fahrzeugen und anderen Straßenverkehrsteilnehmenden bedeutet dies, dass die Inhalte der Kommunikation kontext- und situationsabhängig sind.

\subsection{Wann und wo sollen diese Handlungen ausgeführt werden?}

Das vierte und das fünfte Element zur Herausbildung von kooperative Aktivitäten nach Clark (2006) beziehen sich auf die Ausführung der Handlungen. Es muss eine Einigung darüber getroffen werden, wann und wo die Beteiligten die Inhalte der Kommunikation ausführen. Im Straßenverkehr beziehen sich die auf einer Kommunikation beruhenden Handlungen meist auf (zeitlich) unmittelbar aufeinander folgende Handlungen. Der Ort der Ausführung einer Handlung, das „Wo“ in der Auflistung von Clark (2006), wird im Straßenverkehr von der zugrundeliegenden Infrastruktur der Situation (z.B. einem Zebrastreifen) beeinflusst. Die Einigung über das „Wo“ wird deshalb annahmegemäß unmittelbar über die Art und Weise der Übertragung von Informationen als solches bestimmt (,Wie“). Mit einer wischenden Handgeste kann ein Pkw-Fahrender beispielswei- 
Abb. 1 Gestaltungsdimensionen der Kommunikationsstrategie zwischen automatisierten Fahrzeugen und konventionellen Straßenverkehrsteilnehmenden (Fußgänger/-innen, Fahrrad Fahrende, Pkw-Fahrende, ...) im MTO-Ansatz

Fig. 1 Design dimensions of the communication strategy between automated vehicles and conventional road users (pedestrians, cyclists, vehicle drivers ...) in the MTO-approach

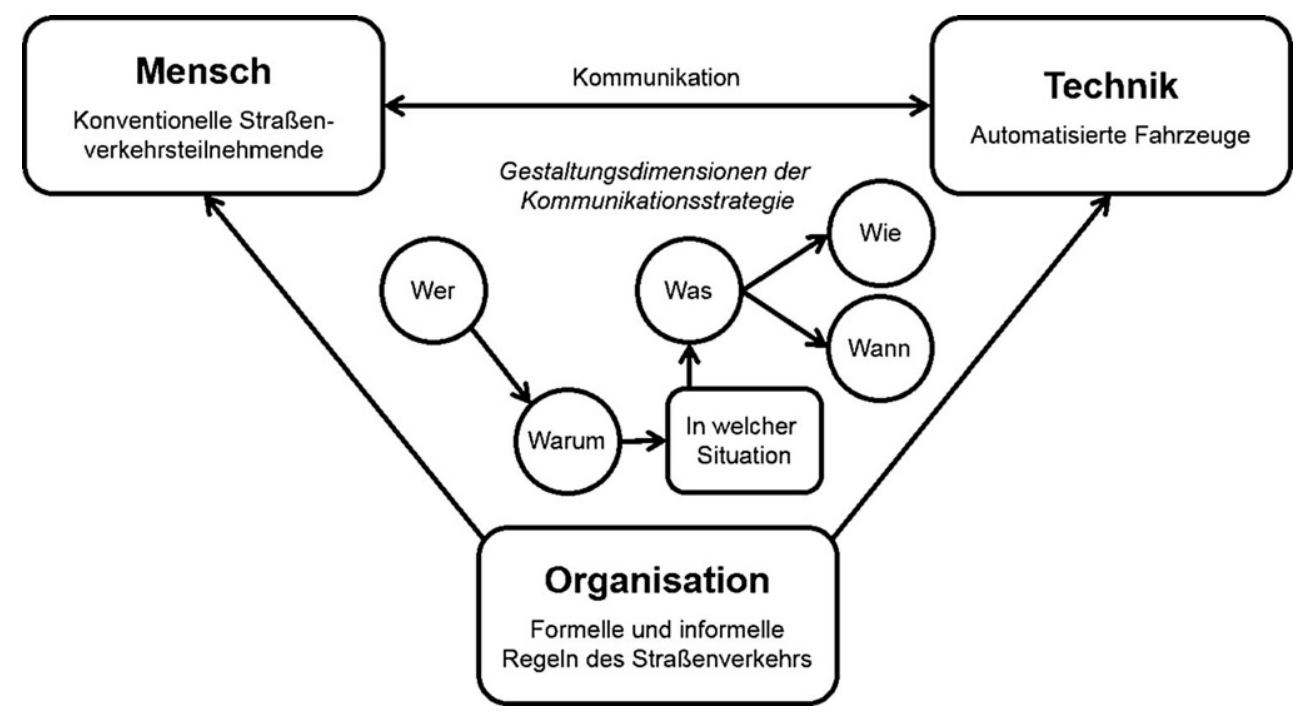

se einen Fußgänger oder eine Fußgängerin dazu auffordern, die Straße (zeitlich unmittelbar) vor dem Pkw zu überqueren (vgl. Färber 2016). Die Ausführung der Handlung, auf die sich die Pkw-und Fußgänger/-in geeinigt haben, wird in diesem Beispiel über eine Handgeste des Pkw-Fahrers initiiert. Neben der Art und Weise der Informationsübertragung stellt sich die zentrale Frage nach dem Zeitpunkt der Kommunikation (,Wann“).

\subsection{Fazit}

Die Einführung von automatisierten Fahrzeugen in den Mischverkehr aus konventionellen und automatisierten Straßenverkehrsteilnehmenden macht es notwendig, Kommunikationsstrategien zwischen Mensch und Technik zu erforschen. Die klassische Kommunikationstheorie bietet Orientierung wie eine Kommunikationsstrategie gestaltet werden sollte, um die Herausbildung von kooperativen Handlungen zwischen den Beteiligten zu fördern. Neben den Beteiligten, Mensch und Technik, nehmen im Kontext des Straßenverkehrs die formellen und informellen Regeln einen bedeutenden, die Kommunikation und Kooperation zwischen Straßenverkehrsteilnehmenden betreffenden, Stellenwert ein. Deshalb eignet sich der Mensch-Technik-Organisation (MTO) Ansatz (vgl. Strohm und Ulich 1997) hier insbesondere als Framework, um die Gestaltungsdimensionen der Kommunikationsstrategie zwischen automatisierten Fahrzeugen und konventionellen Straßenverkehrsteilnehmenden zu gliedern (s. Abb. 1). Das Anwendungsgebiet des Frameworks ist die Technik des automatisierten Fahrzeugs. Die Kommunikation zwischen konventionellen Straßenverkehrsteilnehmenden, also beispielsweise Fußgänger/-innen und Pkw-Fahrenden, wird durch das Framework nicht abgedeckt.
In Abb. 1 ist das Framework dargestellt, das die sechs Gestaltungsdimensionen der Kommunikationsstrategie zwischen konventionellen Straßenverkehrsteilnehmenden und automatisierten Fahrzeugen beinhaltet. Die Dimension „Wer" fragt nach Beteiligten der Kommunikation und legt deren Art und Anzahl, sowie die Kommunikationsrichtung, fest. Aufgrund des zugrundeliegenden Ziels, der Gestaltung von Kommunikation zwischen konventionellen Straßenverkehrsteilnehmenden und automatisierten Fahrzeugen, ist diese Dimension der Kategorie Mensch nah angeordnet. Aus den organisatorischen Rahmenbedingungen des Straßenverkehrs resultieren die Gestaltungsdimensionen „Warum“ und „In welcher Situation“. Diese Dimensionen sind geprägt von der Situation der Kommunikation im Straßenverkehr und spiegeln sich in Zielen und Erwartungen der Beteiligten wider. Daraus leiten sich die Kommunikationsinhalte ab (,Was"). In der Literatur ist diese Gestaltungsdimension vertreten durch das Anzeigen der eigenen Absicht oder dem Auffordern von anderen Straßenverkehrsteilnehmenden (Bazilinskyy et al. 2019). Die Gestaltungsdimension des ,Wie“, also der Art und Weise der Informationsübertragung, ist stark in der Literatur vertreten. Aspekte dieser Gestaltungsdimension beziehen sich auf die Art der Information (implizit oder explizit, vgl. Dey und Terken 2017; Kauffmann et al. 2018), der Modalität der Informationsübertragung (vgl. Colley et al. 2019), der Codierung des Inhalts (vgl. Clerq et al. 2019; Deb et al. 2019; Lee et al. 2019; Löcken et al. 2019a), die Positionierung von Informationen (vgl. Ackermann et al. 2019; Eisma et al. 2020; Liu et al. 2017), die Farbe visueller Informationen (vgl. Bazilinskyy et al. 2019; Reschke et al. 2019; Werner 2018) sowie die Perspektive der Kommunikation (ego- oder allozentrisch, vgl. Bazilinskyy et al. 2019). Die Gestaltungsdimension des "Wann“ beinhaltet die Übertragungszeitpunkte der Kommunikation, 
also der Zeitpunkte, zu denen Kommunikation zwischen automatisierten Fahrzeugen und anderen Straßenverkehrsteilnehmenden stattfindet (vgl. Clerq et al. 2019; Dey et al. 2019). Beide Dimensionen (,Wie“ und „Wann“) sind der Kategorie Technik im MTO Ansatz zugeordnet, weil diese durch die technische Gestaltung des automatisierten Fahrzeugs determiniert werden.

Der Zweck des Frameworks liegt in der Gliederung von Fragegestellungen zur Gestaltung ganzheitlicher Kommunikationsstrategien im Mischverkehr aus konventionellen und automatisierten Straßenverkehrsteilnehmenden. Die Anwendung des Frameworks soll bei der Gestaltung der Kommunikation von automatisierten Fahrzeugen unterstützen, um auch zukünftig die notwendige Kooperation zwischen Straßenverkehrsteilnehmenden sicherzustellen.

Zur Erforschung der Kommunikation zwischen automatisierten Fahrzeugen und konventionellen Straßenverkehrsteilnehmenden werden empirische Studien mit unterschiedlichen methodischen Zugängen durchgeführt (für einen Überblick s. Rasouli und Tsotsos 2020; und Rouchitsas und Alm 2019). Das hergeleitete, theoretische Framework und dessen zugrundeliegenden Fragestellungen können hierbei herangezogen werden, um die aktuell stattfindende Kommunikation strukturiert zu analysieren und daraus dann konkrete technische Gestaltungen der Kommunikation abzuleiten.

\section{Empirische Studien}

Im Folgenden werden zwei empirische Studien beschrieben, die durch die explorative Vorgehensweise weitere Erkenntnisse zur Kommunikation von Fußgänger/-innen mit automatisierten Bussen liefern. Damit adressieren diese Studien die Gestaltungsdimension „Wie“, deren zentrale Fragestellung lautet „Auf welche Art und Weise werden die Inhalte der Kommunikation übertragen?“. Die Untersuchung der Interaktionen mit automatisierten Bussen bietet den Vorteil, dass diese bereits in einigen Testfeldern im realen Straßenverkehr unterwegs sind und somit ein natürliches Interaktionsverhalten zwischen automatisiertem Fahrzeug und Fußgänger/-innen vorliegt.

Die Beteiligten der Kommunikation im Straßenverkehr (Gestaltungsdimension: „Wer") sind in beiden Studien gleich. Ebenso gemeinsam ist die Gestaltungsdimension „In welcher Situation“, die sich hier darstellt als Überquerung der Straße vor dem automatisierten Bus.

\subsection{Forschungsfragen}

Das Ziel beider Studien ist es, Erkenntnisse zur Gestaltung der Kommunikation zwischen Fußgänger/-innen und automatisierten Bussen zu erlangen. Folgende Forschungsfragen stehen dabei im Vordergrund:

- Forschungsfrage 1: Welche Verhaltensweisen von Fußgänger/-innen bei der Interaktion mit automatisierten Bussen kommen vor?

- Forschungsfrage 2: Wie sollen Informationen zur Unterstützung des Entscheidungsverhaltens von Fußgänger/innen bei der Straßenüberquerung vor einem automatisierten Bus aus Sicht von Fußgänger/-innen übertragen werden?

Zur Beantwortung dieser Forschungsfragen wird ein exploratives Vorgehen mit unterschiedlichen methodischen Zugängen gewählt. Die Ergebnisse einer Befragung werden durch Beobachtungen im Feld ergänzt.

\subsection{Methodik}

\subsubsection{Studie 1: Befragung von Fußgänger/-innen}

Zur Beantwortung der Forschungsfragen wurden semistrukturierte Interviews entlang der Strecke und an Bushaltestellen im Testfeld eines automatisierten Busses durchgeführt (Bad Birnbach in Bayern). Die Antworten der Teilnehmenden wurden schriftlich dokumentiert.

Die Befragung war wie folgt aufgebaut: Im ersten Teil wurde der Kenntnisstand und die Erfahrung der Teilnehmenden über das automatisierte Fahren im Allgemeinen und den automatisierten Bus in Bad Birnbach erfasst. Anschließend wurden je der Hälfte der Teilnehmenden eines von zwei Szenarien (A/B) beschrieben, auf das sich die nachfolgenden Fragen beziehen. Diese Szenarien unterscheiden sich durch das Vorhandensein eines Zebrastreifens zur Querung (Szenario A) bzw. durch einen nicht-signalisierten Übergang (Szenario B). Zusätzlich wurde allen Teilnehmenden ein Szenario beschrieben, in dem der Bus den Vorrang gegenüber dem Fußgänger nicht abgeben will (Szenario C). 13 Teilnehmende beantworteten die Fragen im Szenario A, 15 Teilnehmende beantworteten die Fragen in Hinblick auf Szenario B.

Der zweite Teil der Befragung beinhaltete Fragen bezüglich des Entscheidungsverhaltens der Teilnehmenden bei der Straßenüberquerung vor dem automatisierten Bus. Außerdem wurden die Teilnehmenden zu Gestaltungsmöglichkeiten von expliziter Kommunikation befragt, die die Teilnehmenden in dem beschriebenen Szenario bei ihrer Entscheidung unterstützen könnten. Es wurde sowohl nach der Gestaltung eines defensiven Signals (Bus gewährt Fußgänger/-in Vorrang), als auch nach der Gestaltung eines offensiven Signals (Bus möchte Vorrang nicht abgeben) gefragt. Explizite Kommunikation bezieht sich auf die Verwendung von Gesten, Licht- und Tonsignalen zur Kommunikation 
Tab. 2 Beschreibung der Verhaltensweisen von Fußgänger/-innen bei der Interaktion mit dem automatisierten Bus (Madigan et al. 2019; Übersetzung der Verfasser)

Table 2 Description of the interaction scenarios of pedestrian when interacting with the automated bus (Madigan et al. 2019; translation by the authors)

\begin{tabular}{ll}
\hline Verhaltensweise & Beschreibung der Verhaltensweisen (Subkategorie) \\
\hline (1) Straßenüberque- & Fußgänger/-in erhöht seine/ihre Geschwindigkeit, um vor dem Bus die Straße zu überqueren (Blick auf den Bus) \\
& Fußgänger/-in erhöht seine/ihre Geschwindigkeit, um vor dem Bus die Straße zu überqueren (Blick nicht auf den Bus) \\
& Fußgänger/-in behält seine/ihre Geschwindigkeit bei, während er/sie vor dem Bus die Straße überquert (Blick auf den \\
& Bus) \\
& Fußgänger/-in behält seine/ihre Geschwindigkeit bei, während er/sie vor dem Bus die Straße überquert (Blick auf den \\
& Bus) \\
& Fußgänger/-in bewegt sich in der selben Richtung wie der Bus (rechtseitig) \\
(2) Bewegung parallel & Fußgänger/-in bewegt sich in der entgegengesetzten Richtung zum Bus (rechtsseitig) \\
zum Bus & Fußgänger/-in bewegt sich in der selben Richtung wie der Bus (linksseitig) \\
& Fußgänger/-in bewegt sich in der entgegengesetzten Richtung zum Bus (linksseitig) \\
& Fußgänger/-in ändert die Trajektorie seiner/ihrer Bewegung durch das Betreten und das darauffolgende Zurückwei- \\
(3) Änderung der & Fußgänger/-in ändert die Trajektorie seiner/ihrer Bewegung durch Ausweichen der Bewegungsbahn des Busses \\
Bewegungsrichtung & Fußgänger/-in hält an, um den Bus vorbei zu lassen, obwohl er/sie Vorfahrt hat \\
(4) Stoppen, um Bus & Fußgänger/-in hält an, um den Bus vorbei zu lassen, der Vorfahrt hat \\
vorbeizulassen & Fußgänger/-in hält an, um den Bus vorbei zu lassen; die Vorfahrt ist nicht eindeutig geklärt \\
& Fußgänger/-in testet den Bus indem er/sie in die Bewegungsbahn des Busses tritt \\
(5) „Testen“ des & Fußgänger/-in testet den Bus indem er/sie im letzten Moment die Bewegungsbahn des Busses verlässt \\
automatisierten Busses &
\end{tabular}

zwischen Straßenverkehrsteilnehmenden (Imbsweiler et al. 2018a).

Zum Abschluss der Befragung wurden demografische Daten der Teilnehmenden dokumentiert. Aufgenommen wurden Geschlecht und Alter (vier Altersgruppen: <20 Jahre, 20-40 Jahre, 41-60 Jahre, >60 Jahre).

Die Interviews wurden transkribiert und qualitativ ausgewertet. Die Antworten der Teilnehmenden wurden zum Zwecke der Vergleichbarkeit kategorisiert.

Die Gesamtstichprobe betrug 30 Teilnehmende. Das Kollektiv der Teilnehmenden bestand aus zehn Männern und 19 Frauen. Über die Hälfte der Teilnehmenden (16) war 61 Jahre oder älter. Sechs Teilnehmende waren zwischen 41 und 60 Jahre alt. Sieben Teilnehmende wurden der Altersgruppe der 20 bis 40-jährigen zugeordnet. Die Altersgruppe der unter 20-jährigen war nicht vertreten. Die demografischen Angaben einer Person fehlen in den Aufzeichnungen.

Von insgesamt 30 Teilnehmenden wussten 28, dass es einen automatisierten Bus in Bad Birnbach gibt. Alle Teilnehmenden, die von der Existenz des automatisierten Busses wussten, hatten diesen auch schon einmal gesehen. Zwölf Teilnehmende aus dieser Gruppe sind schon mindestens einmal mit dem Bus gefahren. Zwei Interviews mit Teilnehmenden, die nichts über die Existenz des automatisierten Busses in Bad Birnbach wussten, wurden ausgeschlossen.

\subsubsection{Studie 2: Beobachtung von Fußgänger/-innen}

Ergänzend wurde eine Beobachtungsstudie als explorative Feldbeobachtung von Interaktionen zwischen Fußgänger/innen und automatisierten Bussen in Frankfurt am Main durchgeführt. Untersuchungsgegenstand war das beobachtbare Verhalten von Fußgänger/-innen. Des Weiteren wurde das Geschlecht der beobachteten Personen sowie die zugrundeliegende Situation der Beobachtung protokolliert.

Der Beobachtungsort war eine Teststrecke in Frankfurt am Main. Die Teststrecke ist für sonstige motorisierte Fahrzeuge (ausgenommen Lieferverkehr) gesperrt. Die Fahrspur besteht aus zwei Fahrsteifen, auf denen der Bus fährt, und anliegenden Fußgängerwegen. Fünf Situationen wurden entlang der Fahrtstrecke des automatisierten Busses unterschieden:

- Die Fahrt auf einem 150 m langen, gerade Streckenabschnitt (a)

- Eine ehemalige Lichtsignalanlage für Fußgänger/-innen, die aufgrund der Sperrung der Teststrecke abgehängt wurde; Straßenmarkierungen und Verkehrszeichen sind noch sichtbar (b)

- Die Wendezone des automatisierten Busses; Der Bus setzt hier zurück, um zu wenden (c)

- Nahe einer Haltestelle des automatisierten Busses (d)

- Ein Bereich, der als Zubringerweg für Fußgänger/-innen direkt auf die Mainuferpromenade führt (e) 
Tab. 3 Kategorien des Entscheidungsverhaltens von Fußgänger/innen im Kontakt mit dem automatisierten Bus (Anzahl der Nennungen nach Szenario und gesamt; $N=28$ )

Table 3 Categories of decision behaviour of pedestrians in contact with the automated bus (number of mentions by scenario and total; $N=28$ )

\begin{tabular}{|c|c|c|c|c|c|c|}
\hline \multirow[t]{3}{*}{ Entscheidungsverhalten } & \multicolumn{4}{|c|}{ Szenario } & \multirow{3}{*}{\multicolumn{2}{|c|}{ Gesamt }} \\
\hline & \multicolumn{2}{|l|}{$\mathrm{A}$} & \multicolumn{2}{|l|}{$\mathrm{B}$} & & \\
\hline & \multicolumn{2}{|c|}{ (Zebrastreifen) } & \multicolumn{2}{|c|}{ (Nicht-signalisierter Übergang) } & & \\
\hline Stehen bleiben, warten & 4 & $(31 \%)$ & 11 & $(73 \%)$ & 15 & $(54 \%)$ \\
\hline Gehen, nicht warten & 5 & $(38 \%)$ & 4 & $(27 \%)$ & 9 & $(32 \%)$ \\
\hline Abhängig vom Verhalten des Busses & 4 & $(31 \%)$ & - & & 4 & $(14 \%)$ \\
\hline Gesamt & 13 & $(100 \%)$ & 15 & $(100 \%)$ & 28 & $(100 \%)$ \\
\hline
\end{tabular}

Anmerkung: Die Prozentangaben beziehen sich auf die Spaltensumme

Die Beobachtungen wurden, unter zu Hilfenahme eines Tablets, direkt protokolliert. Insgesamt wurde $15 \mathrm{~h}$ beobachtet, im Zeitraum zwischen dem 4. und 10. Dezember 2019.

Das Ereignis, welches ein Aufzeichnen der Beobachtung auslöste, wurde wie folgt definiert: Situationen, in denen die Beteiligten der Interaktion ihr Verhalten vor einer potentiellen „Konfliktzone“ anpassen, sodass Zeit und Raum für fließende Bewegungen zwischen beiden Straßenverkehrsteilnehmenden bleibt (Cloutier et al. 2017). Dies bedeutet, dass Interaktionen protokolliert wurden, bei denen Fußgänger/-innen in den relevanten Nahbereich des Buses eintreten, so dass dieser von seinem regulären Fahrtprotokoll abweichen muss oder Situationen, in denen Fußgänger/-innen ihr Verhalten ändern, z.B. stehen bleiben. Der für die Interaktion relevante Nahbereich umfasste situationsabhängig zwischen 0 und 15 Metern radial zum Fahrzeug.

Zur Beschreibung der Interaktionen wurde auf die Erkenntnisse von Madigan et al. (2019) zurückgegriffen, die eine Klassifikation von beobachtbaren Verhaltensweisen von Fußgänger/-innen bei der Interaktion mit automatisierten Bussen liefern. Es werden fünf Verhaltensweisen von Fußgänger/innen unterschieden: (1) die Straßenüberquerung vor dem Bus, (2) die Bewegung parallel zum Bus, (3) die Änderung der Bewegungsrichtung, (4) das Stoppen, um den Bus vorbeizulassen und (5) das Testen des automatisierten Busses. Die Verhaltensweisen werden in Tab. 2 beschrieben.

Ausgeschlossen von der Beobachtung wurden an Haltestellen wartende Personen und Personen, die aus dem Fahrzeug aussteigen. Insgesamt wurden 308 Interaktionen im Beobachtungszeitraum protokolliert. Davon konnten 264 Interaktionen eindeutig der o.g. Klassifikation nach Madigan et al. (2019) zugeordnet werden.

\subsection{Ergebnisse}

\subsubsection{Studie 1: Befragung von Fußgänger/-innen}

Auf die Frage, wie sich die Teilnehmenden entscheiden würden, ob sie die Straße bei einem Zusammentreffen mit dem automatisierten Bus vor diesem überqueren würden, antworteten insgesamt 15 Teilnehmende, dass sie stehen bleiben würden. Von diesen Teilnehmenden gaben sieben an, auf jeden Fall hinter dem Bus zu überqueren, weil sie der Technik des Busses nicht vertrauen würden. Die verbleibenden acht Teilnehmenden würden warten und die Straße erst überqueren, wenn der Bus zum Stehen gekommen ist. Weitere neun Teilnehmende gaben an, dass sie, ohne zu warten, vor dem Bus die Straße queren würden. Diese Teilnehmenden begründeten ihr geplantes Verhalten durch das Vertrauen in die Technik des automatisierten Busses. Vier Teilnehmende machten ihre Entscheidung abhängig vom Verhalten des Busses. Als wesentliche Einflussvariablen nannten die Probanden die Geschwindigkeit und die Entfernung des Busses.

Die Antworten der Teilnehmenden in Szenario A und B unterscheiden sich vor allem in der Kategorie „Stehen bleiben“. Während vier Teilnehmende in Szenario A (Zebrastreifen) auf jeden Fall stehen bleiben und warten würden, entschieden sich elf Teilnehmende in Szenario B (nichtsignalisierter Übergang) für dieses Verhalten. Die Anzahl der Nennungen des Entscheidungsverhaltens von Fußgänger/-innen im Kontakt mit dem automatisiert fahrenden Bus sind in Tab. 3 zusammengefasst.

Die Teilnehmenden wurden nach Gestaltungslösungen expliziter Kommunikation, die sie bei dem beschriebenen Szenario unterstützen könnten, gefragt. Es konnten mehrere Gestaltungsvorschläge abgeben werden. Die Ergebnisse sind in Tab. 4 zusammengefasst.

Auf die Frage nach defensiven Signalen des Busses (der Bus gewährt Vorrang) nannten die Teilnehmenden am häufigsten Symbole $(n=8)$. Diese umschrieben die Teilnehmenden als „Ampelsymbol“, „Bild“ oder „Zeichen“. Die Gestaltungsvorschläge „Schrift" und „akustisches Signal“ wurden jeweils sieben Mal genannt, gefolgt vom Gestaltungsvorschlag „Licht“ mit 5 Nennungen. Die Farbe Grün wurde von den Teilnehmenden damit verknüpft, dass sie die Straße vor dem Bus überqueren können. Konkretisiert wurde der Gestaltungsvorschlag „Schrift“ über bestimmte Texte (beispielsweise "Sie können laufen“) oder einer Countdown-Anzeige (,Fährt weiter in ... Sekunden“). Des 
Tab.4 Gestaltungsvorschläge expliziter Kommunikation des automatisierten Busses (Anzahl der Nennungen für defensive und offensive Signale; Mehrfachnennungen möglich; $\mathrm{M}=72 ; N=28$ )

Table 4 Design proposals for explicit communication of the automated bus (number of mentions by scenario and total; multiple mentions possible; $\mathrm{M}=72 ; N=28$ )

\begin{tabular}{|c|c|c|c|c|}
\hline \multirow{3}{*}{$\begin{array}{l}\text { Gestaltungsvorschlag (explizite } \\
\text { Kommunikation) }\end{array}$} & \multicolumn{4}{|c|}{ Art des Signals } \\
\hline & \multicolumn{2}{|c|}{ Defensiv } & \multicolumn{2}{|c|}{ Offensiv } \\
\hline & \multicolumn{2}{|c|}{ (Bus gewährt Vorrang) } & \multicolumn{2}{|c|}{ (Bus gewährt keinen Vorrang) } \\
\hline Schrift & 7 & $(20,5 \%)$ & 4 & $(11 \%)$ \\
\hline Licht & 5 & $(15 \%)$ & 2 & $(5 \%)$ \\
\hline Symbol & 8 & $(23 \%)$ & 2 & $(5 \%)$ \\
\hline Akustisches Signal & 7 & $(20,5 \%)$ & 20 & $(53 \%)$ \\
\hline Anthropomorphismus & 5 & $(15 \%)$ & 2 & $(5 \%)$ \\
\hline Links Blinken & - & & 7 & $(18 \%)$ \\
\hline Keine Information & 2 & $(6 \%)$ & 1 & $(3 \%)$ \\
\hline Gesamt & 34 & $(100 \%)$ & 38 & $(100 \%)$ \\
\hline
\end{tabular}

Weiteren wurden anthropomorphe Gestaltungen, wie beispielsweise menschliche Gestik, Sprache und Augenzwinkern, genannt. Zwei Teilnehmende gaben an, dass keine zusätzlichen Informationen nötig sind. Acht Teilnehmende gaben mehr als einen Gestaltungsvorschlag ab.

Die Ergebnisse hinsichtlich offensiver Kommunikationssignale (Bus gewährt keinen Vorrang) zeigen, dass das akustische Signal mit 20 Nennungen mit Abstand am häufigsten angegeben wurde. Das akustische Signal umschrieben die Teilnehmenden mit einem Ton beim Anfahren des Busses (,ähnlich wie bei einer Straßenbahn“) oder einem Hupen. Als offensives Signal nannten die Teilnehmenden zudem das „Links Blinken“. Die Gestaltungsvorschläge „Schrift“, „Symbol“ (Stoppzeichen), und „Anthropomorphismus“ (Sprachausgabe) wurden nur vereinzelt genannt. Eine Person gab an, dass sie keine zusätzlichen Informationen in dieser Situation benötigt.

\subsubsection{Studie 2: Beobachtung von Fußgänger/-innen}

Die beobachteten Verhaltensweisen von Fußgänger/-innen bei der Interaktion mit automatisierten Bussen wurden der Klassifikation nach Madigan et al. (2019) zugeordnet (s. Tab. 5). Die am häufigsten beobachtete Verhaltensweise ist die Straßenüberquerung vor dem Bus (124 Beobachtungen; $47 \%$ aller beobachteten Interaktionen), gefolgt von der Bewegung von Fußgänger/-innen parallel zum Bus (61; $23 \%$ ) und dem Stoppen von Fußgänger/-innen, um den Bus vorbeizulassen $(54 ; 20,5 \%)$. Seltener beobachtete Verhaltensweisen sind die Änderung der Bewegungsrichtung von Fußgänger/-innen als Reaktion auf den automatisierten Bus (20; 7,5\%) und das „Testen“ des automatisierten Busses durch Fußgänger/-innen $(5 ; 2 \%)$.

Die Detailanalyse der Interaktionen entsprechend der in Tab. 2 dargestellten Subkategorien ist im Folgenden dargestellt.
Für den Interaktionstyp ,Straßenüberquerung vor dem $B u s$ “ zeigt sich, dass Fußgänger/-innen am häufigsten mit gleichbleibender Geschwindigkeit die Straße vor dem Bus überqueren (99 von 124 Interaktionen). Bei 24 von 124 Interaktionen wird beobachtet, dass die Fußgängerin bzw. der Fußgänger schneller wird während der Straßenüberquerung vor dem automatisierten Bus. Eine Blickzuwendung zum automatisierten Bus bei der Straßenüberquerung von Fußgänger/-innen wird in 88 von 124 Interaktionen beobachtet.

Die Bewegung parallel zum Bus ist der am zweithäufigsten beobachtete Interaktionstyp. Die beobachteten Verhaltensweisen teilen sich gleichmäßig auf Interaktionen in entgegengesetzter oder selber Richtung und nah der rechten oder linken Seite des automatisierten Busses auf.

Die Detailanalyse des Interaktionstyps ,Änderung der Bewegungsrichtung “ zeigt, dass Fußgänger/-innen bei einer Änderung der Bewegungsrichtung vor allem mit einem ausweichen, also dem Vermeiden von Kontakt mit dem automatisierten Bus, reagieren (16 von 20 Interaktionen). Es können vier Interaktionen beobachtet werden, in denen die Änderung der Bewegungsrichtung in einem Eintreten und ein darauffolgendes, sofortiges Verlassen der Fahrbahn des automatisierten Busses besteht.

In insgesamt fünf Fällen wird ein „Testen“ des automatisierten Busses durch Fußgänger beobachtet. Drei Fußgänger testen den Bus, indem sie in seine Fahrbahn treten. Zwei Fußgänger gehen dem automatisierten Bus erst im letzten Moment aus dem Weg und verlassen die Fahrbahn.

Um den Einfluss von kontextuellen Variablen $\mathrm{zu}$ testen, werden die beobachteten Verhaltensweisen in Abhängigkeit der Situation analysiert (s. Tab. 5). Die Verhaltensweisen der beobachteten Personen und die Situation stehen in einem Zusammenhang (Exakter Test nach Fisher $=68.281, p=0,000)$. Der Zusammenhang ist nach Cohen (1988) stark ausgeprägt $\left(C_{k o r r}=0,506, p=0,000\right.$; Cramers $V=0,254, p=0,000)$. 
Tab. 5 Beobachtete Verhaltensweisen von Fußgänger/-innen bei der Interaktion mit dem automatisierten Bus $(N$ gesamt $=264)$

Table 5 Observed Behaviour of pedestrians when interacting with the automated bus $(N$ total $=264)$

\begin{tabular}{|c|c|c|c|c|c|c|c|c|c|c|c|c|}
\hline \multirow{3}{*}{$\begin{array}{l}\text { Beobachtete } \\
\text { Verhaltensweisen }\end{array}$} & \multicolumn{10}{|c|}{ Situation } & \multirow{3}{*}{\multicolumn{2}{|c|}{ Gesamt }} \\
\hline & \multicolumn{2}{|l|}{$\mathrm{a}$} & \multicolumn{2}{|l|}{$b$} & \multicolumn{2}{|l|}{$\mathrm{c}$} & \multicolumn{2}{|l|}{$\mathrm{d}$} & \multicolumn{2}{|l|}{$\mathrm{e}$} & & \\
\hline & \multicolumn{2}{|c|}{ Gerade Strecke } & \multicolumn{2}{|c|}{$\begin{array}{l}\text { Ehemalige Lichtsi- } \\
\text { gnalanlage }\end{array}$} & \multicolumn{2}{|c|}{ Wendezone } & \multicolumn{2}{|c|}{ Haltestelle } & \multicolumn{2}{|c|}{ Zubringerweg } & & \\
\hline $\begin{array}{l}\text { Straßenüberquerung } \\
\text { vor dem Bus }\end{array}$ & 8 & $(16 \%)$ & 38 & $(55 \%)$ & 22 & $(45 \%)$ & 44 & $(70 \%)$ & 12 & $(36 \%)$ & 124 & $(47 \%)$ \\
\hline $\begin{array}{l}\text { Bewegung parallel } \\
\text { zum Bus }\end{array}$ & 20 & $(40 \%)$ & 5 & $(7,25 \%)$ & 14 & $(29 \%)$ & 15 & $(24 \%)$ & 7 & $(21 \%)$ & 61 & $(23 \%)$ \\
\hline $\begin{array}{l}\text { Änderung der Be- } \\
\text { wegungsrichtung }\end{array}$ & 3 & $(6 \%)$ & 5 & $(7,25 \%)$ & 7 & $(14 \%)$ & 3 & $(4,5 \%)$ & 2 & $(6,5 \%)$ & 20 & $(7,5 \%)$ \\
\hline $\begin{array}{l}\text { Stoppen, um Bus } \\
\text { vorbeizulassen }\end{array}$ & 19 & $(38 \%)$ & 18 & $(26 \%)$ & 6 & $(12 \%)$ & 1 & $(1,5 \%)$ & 10 & $(30 \%)$ & 54 & $(20,5 \%)$ \\
\hline $\begin{array}{l}\text { „Testen“ des auto- } \\
\text { matisierten Busses }\end{array}$ & - & & 3 & $(4,5 \%)$ & - & & - & & 2 & $(6,5 \%)$ & 5 & $(2 \%)$ \\
\hline Gesamt & 50 & $(100 \%)$ & 69 & $(100 \%)$ & 49 & $(100 \%)$ & 63 & $(100 \%)$ & 33 & $(100 \%)$ & 264 & $(100 \%)$ \\
\hline
\end{tabular}

Anmerkung: Die Prozentangaben beziehen sich auf die Spaltensumme

\section{Diskussion}

\subsection{Gestaltungsrahmen der Interaktion zwischen automatisierten Fahrzeugen und anderen Straßenverkehrsteilnehmenden}

Der Straßenverkehr ist ein sozialer Raum, in dem Menschen fortlaufend miteinander interagieren. Die geplante Einführung von automatisierten Fahrzeugen in den urbanen Straßenverkehr ist diesbezüglich mit Chancen und Risiken verbunden, die es zu erforschen gilt. Die Chancen liegen in einer erhöhten Sicherheit für andere Straßenverkehrsteilnehmende, die in einer defensiven und gesetzeskonformen Fahrweise von automatisierten Fahrzeugen begründet ist (Millard-Ball 2018), sowie einer erhöhten Effizienz des Straßenverkehrsflusses (Fagnant und Kockelman 2015). Ein Risiko besteht in möglichen Verhaltensanpassungen von Straßenverkehrsteilnehmenden, wie beispielsweise das vermehrte, unachtsame Betreten einer Straße durch Fußgänger/-innen aufgrund eines Übervertrauens in die Sensorik und Aktorik von automatisierten Fahrzeugen (Millard-Ball 2018). Um die Sicherheit bei der Teilnahme automatisierter Fahrzeuge am Straßenverkehr zu maximieren und die Risiken zu minimieren werden natürliche Interaktionen, die sich nahtlos in den heuten Straßenverkehr einfügen, zwischen automatisierten Fahrzeugen und anderen Straßenverkehrsteilnehmenden angestrebt. Dazu werden viele heterogene Kommunikationskonzepte entwickelt und erforscht (für einen Überblick s. Schieben et al. 2019; Bazilinskyy et al. 2019).

Zur Analyse der Kommunikation zwischen automatisierten Fahrzeugen und anderen Straßenverkehrsteilnehmenden wurden in diesem Beitrag, angelehnt an die Kommunikationstheorie nach Clark (2006), Gestaltungsdimensionen abgeleitet. Die Einbettung dieser Gestaltungsdimensionen in einem Framework nach dem MTO-Ansatz zeigt die zugrundeliegenden multiplen Dimensionen auf und legt die damit einhergehende Komplexität der Problemstellung offen. Das Framework soll bei der Gestaltung der Kommunikation von automatisierten Fahrzeugen unterstützen und so auch zukünftig die notwendige Kooperation zwischen Straßenverkehrsteilnehmenden durch Kommunikation sicherstellen.

Aus der Betrachtung einzelner Gestaltungsdimensionen können einzelne Fragestellungen abgeleitet werden. Charakteristisch für den urbanen Verkehrsraum ist die Vielzahl und Heterogenität von Straßenverkehrsteilnehmenden (Gestaltungsdimension: „Wer"). Die Kommunikationsstrategie eines automatisierten Fahrzeugs muss sicherstellen, dass sie gerichtet kommuniziert, um Missverständnissen vorzubeugen. Unterschiedliche Straßenverkehrsteilnehmende stellen spezifische Anforderungen an die Kommunikation mit automatisierten Fahrzeugen. Beim Anbringen von Displays auf der Karosserie eines automatisierten Fahrzeugs müssen beispielsweise die Sichthöhen unterschiedlicher Straßenverkehrsteilnehmender berücksichtigt werden (vgl. Colley et al. 2019).

Straßenverkehrsteilnehmende nehmen im Straßenverkehr unterschiedliche Rollen ein (z.B. den Vorrang betreffend; Gestaltungsdimension: „Warum“). Die Interpretation dieser Rollen erlaubt ihnen eine effiziente Kommunikation. Offen bleibt die Forschungsfrage, ob automatisierten Fahrzeugen im Straßenverkehr eine besondere Rolle zugeschrieben wird. Erste Erkenntnisse liefert hier die Akzeptanzforschung von automatisierten Fahrzeugen (vgl. Penmetsa et al. 2019).

Von herausragender Bedeutung für das Zustandekommen von Kommunikation im Straßenverkehr und die Interpretation der spezifischen Rollen von Straßenverkehrsteilnehmenden ist die Situation der Kommunikation (Gestaltungsdimension: „In welcher Situation“). Die Organi- 
sation im Straßenverkehr nimmt hierbei Einfluss auf die Art und Weise der Kommunikation (Gestaltungsdimension: „Wie“). Vieles deutet darauf hin, dass in Situationen, die formellen Regeln unterliegen, eine implizite Kommunikation, z.B. durch Abbremsen des Fahrzeugs, ausreicht, während in informell geregelten Situationen, z.B. bei einem Deadlock, stärker explizit kommuniziert werden muss (vgl. Ackermann et al. 2019).

\subsection{Interaktionsverhalten von Fußgänger/ -innen mit automatisierten Fahrzeugen und Rückschlüsse auf die Gestaltung der Kommunikationsstrategie}

Zur Entwicklung holistischer Kommunikationsstrategien zwischen automatisierten Fahrzeugen und anderen Straßenverkehrsteilnehmenden müssen alle Dimensionen der Kommunikationsstrategie zwischen Straßenverkehrsteilnehmenden adressiert werden. In diesem Beitrag wird die Dimension „Wie“ (,Auf welche Art und Weise werden die Informationen übertragen?") anhand zweier empirischer Studien untersucht.

Die Befragung von Fußgänger/-innen zum Entscheidungsverhalten bei der Straßenüberquerung vor dem automatisierten Bus zeigt einen starken Einfluss der Situation (Zebrastreifen vs. nicht-signalisierter Übergang). Mit der Abnahme formeller Regeln im Straßenverkehr dominiert die Strategie des „Stehen bleiben, warten“ auf Seiten der Fußgänger/-innen. Dies spiegelt die Ergebnisse von Merat et al. (2018) wider, die zeigen, dass Fußgänger/-innen bei der Interaktion mit automatisierten Bussen mehr Informationen benötigen, wenn Straßenmarkierungen fehlen. Als weitere wichtige Einflussvariable des Entscheidungsverhaltens von Fußgänger/-innen konnte das Vertrauen in die Automation identifiziert werden (vgl. Wintersberger et al. 2019). Teilnehmende der Befragung mit der Strategie „Gehen, nicht warten" berichten, dass sie dem automatisierten Bus vertrauen würden. Beim Aufbau dieses Vertrauens spielen vergangene Interaktionen mit dem automatisierten Bus eine entscheidende Rolle (vgl. Penmetsa et al. 2019). Des Weiteren deuten die Teilnehmenden der Befragung an, dass implizite Kommunikation (Geschwindigkeit des Fahrzeugs, Abstand zum Fahrzeug) für ihre Entscheidungsbildung wichtig ist (vgl. Beggiato et al. 2017).

Die verdeckte Beobachtung von Fußgänger/-innen im Testfeld eines automatisierten Busses in Frankfurt am Main ergänzen die Ergebnisse der Befragung. Die beobachteten Verhaltensweisen konnten der Klassifikation nach Madigan et al. (2019) zugeordnet werden. Im Gegensatz zu den Ergebnissen von Madigan et al. (2019) wurde das Stoppen vor dem automatisierten Bus, um diesen vorbeizulassen, häufiger beobachtet. Dies lässt darauf schließen, dass die beobachteten Personen risiko-avers mit dem automatisierten Bus interagieren. Trotzdem lässt der hohe prozentuale Anteil an Straßenüberquerung darauf schließen, dass Fußgänger/-innen dem automatisierten Bus nicht übermäßig misstrauen. Dies deckt sich mit den Ergebnissen der Befragung von Fußgänger/-innen in Bad Birnbach.

Auch konnte ein „Testen“ des automatisierten Busses durch Fußgänger beobachtet werden. Dieses Testen deutet auf Verhaltensanpassungen von Fußgänger/-innen hin (vgl. Abschn. 4.1) und begründet neue Anwendungsfälle der Kommunikation zwischen Straßenverkehrsteilnehmenden. Verhaltensanpassungen, ausgelöst durch ein Übervertrauen in die defensive Reaktion von automatisierten Fahrzeugen, könnten dem Ziel einer gesteigerten Sicherheit von Fußgänger/-innen und Effizienz im Straßenverkehr entgegenstehen.

Der Vergleich beider Studien zeigt, dass das beobachtbare Verhalten von Fußgänger/-innen tendenziell von dem subjektiv erfragten Verhalten abweicht. Während eine risiko-averse Strategie (,Stehen bleiben, warten“) von mindestens einem Drittel der befragten Fußgänger/-innen gewählt wird, zeigt die Beobachtung von Fußgänger/-innen im Feld, dass eine Vielzahl von Verhaltensweisen beobachtet werden können, die insgesamt keine Rückschlüsse auf eine Dominanz von risiko-aversem Verhalten zulassen. Aufgrund vorliegender Unterschiede der empirischen Studie, bspw. hinsichtlich Studienkollektiv und Ort der Durchführung, stellt dieses Ergebnis einen vorläufigen Befund dar, der weiterer Erforschung bedarf.

Weitere Limitationen der empirischen Studien sind im Folgenden beschreiben: Mittels der Befragung im Testfeld Bad Birnbach konnten Menschen erreicht werden, die in der Vergangenheit mit dem automatisierten Bus interagierten. Die Altersverteilung der Teilnehmenden an der Befragung ist rechtssteil. Jüngere Altersgruppen $(<20$ Jahre $)$ sind nicht vertreten. Im Zuge eines „Design-for-All“ Ansatzes müssen in folgenden Untersuchungen weitere Gruppen, wie bspw. Kinder (vgl. Charisi et al. 2017) und Menschen mit Leistungseinschränkungen (vgl. Colley et al. 2019), berücksichtigt werden. Die Ergebnisse der empirischen Studien erlauben grundsätzliche Rückschlüsse auf das zu erwartende Verhalten von Fußgänger/-innen bei der Interaktion mit automatisierten Fahrzeugen. Aufgrund der begrenzten Studienkollektive und der zugrundeliegenden Datenbasis handelt es sich dabei um Trends, die durch thesenunterstützte Forschungen weitergehend ergründet werden müssen. Auf eine tiefergehende Analyse der beobachtbaren Verhaltensweisen mittels Videoaufnahmen wurde aus rechtlichen Gründen des Datenschutzes verzichtet. Der Einsatz von Videoaufnahmen sollte für zukünftige Untersuchungen geprüft werden.

Aus beiden Studien können Rückschlüsse auf die Gestaltung der Kommunikationsstrategie zwischen Fußgänger/-innen und automatisierten Bussen gewonnen werden: 
Die Befragung von Fußgänger/-innen zeigt in Bezug auf die Gestaltungsdimension „Wie“, dass verschiedene Arten von Signalen (Schrift, Ton, Symbole, ...) zur Unterstützung des Entscheidungsverhaltens von Fußgänger/-innen bei der Querung vor dem Bus beitragen könnten (vgl. hierzu auch Löcken et al. 2019b; Verma et al. 2019). Für den Entwurf und die Entwicklung holistischer Kommunikationsstrategien im Straßenverkehr sollten deshalb zur Ergänzung impliziter Kommunikation auch explizite Kommunikationssignale berücksichtigt werden. Hinsichtlich der Unterscheidung von defensiven und offensiven Signalen wird deutlich, dass die Probanden akustische Signale am häufigsten mit einem offensiven Signal in Verbindung bringen. Eine multimodale Gestaltung von expliziten Kommunikationssignalen ist vielversprechend.

Hinsichtlich der Gestaltung von Kommunikationsstrategien zwischen Fußgänger/-innen und automatisierten Bussen zeigt sich, dass bei $29 \%$ aller Straßenüberquerungen vor dem automatisierten Bus kein Blick in Richtung des Busses von Fußgänger/-innen beobachtet wurde. Fußgänger/-innen suchen nicht in jedem Fall der Straßenüberquerung den Blickkontakt mit Fahrzeuginsassen (vgl. Dey und Terken 2017). Eine Gestaltung der Kommunikationssignale von automatisierten Bussen rein über den visuellen Sinneskanal unterstützt nicht das natürliche Interaktionsverhalten aller Fußgänger/-innen.

Ein zentrales Ergebnis der Beobachtungsstudie ist die Situationsabhängigkeit der beobachteten Verhaltensweisen von Fußgänger/-innen. Hierbei bestätigt sich die Annahme des postulierten Frameworks, dass sich das Verhalten und daraus abgeleitet auch der Kommunikationsbedarf von Straßenverkehrsteilnehmenden in Abhängigkeit von der Situation unterscheidet. Für die Gestaltung der Kommunikation bedeutet dies, dass sich Kommunikationskonzepte in unterschiedlichen Situation bewähren müssen. Bei der Gestaltung der Kommunikation zwischen automatisierten Fahrzeugen und anderen Straßenverkehrsteilnehmenden sollten auch Anwendungsfälle von „testenden“ Fußgänger/-innen berücksichtigt werden. Weitere Forschung ist nötig, um den Effekt von technischen Kommunikationssignalen von automatisierten Fahrzeugen auf mögliche Verhaltensanpassungen zu untersuchen.

\section{Fazit}

Die Integration von automatisierten Fahrzeugen in den Mischverkehr aus automatisierten und konventionellen Straßenverkehrsteilnehmenden verlangt nach der Gestaltung von Kooperation und Kommunikation zwischen den Beteiligten. Die Gestaltungsdimensionen der Kommunikationsstrategie zwischen automatisierten Fahrzeugen und konventionellen Straßenverkehrsteilnehmenden im MTO-
Ansatz dient der Analyse oder strukturierten und vollständigen Gestaltung zukünftiger Kommunikationsstrategien. Neben der Art und Weise der Kommunikation sollten zukünftig auch übergeordnete Gestaltungsdimensionen, vor allem die Art und Anzahl der Beteiligten und deren eingenommene Rollen, stärkere Berücksichtigung finden. Weiterer Untersuchungsbedarf besteht zum Beispiel hinsichtlich des Gruppenverhaltens von Fußgänger/-innen und der Möglichkeit der gerichteten Kommunikation mit Einzelnen. Die Erforschung des Interaktionsverhalten von Straßenverkehrsteilnehmenden mit automatisierten Fahrzeugen bildet die Basis für die Integration von spezifischen Kommunikationsstrategien in automatisierte Fahrzeuge.

Danksagung Die vorliegende Arbeit ist ein Ergebnis des Verbundforschungsprojekts @CITY - Automated Cars and Intelligent Traffic in the City. Das Forschungsvorhaben wird vom Bundesministerium für Wirtschaft und Energie (BMWi) aufgrund eines Beschlusses des Deutschen Bundestages gefördert. Die Verantwortung für den Inhalt dieser Veröffentlichung trägt allein der Verfasser.

Funding Open Access funding provided by Projekt DEAL.

Open Access Dieser Artikel wird unter der Creative Commons Namensnennung 4.0 International Lizenz veröffentlicht, welche die Nutzung, Vervielfältigung, Bearbeitung, Verbreitung und Wiedergabe in jeglichem Medium und Format erlaubt, sofern Sie den/die ursprünglichen Autor(en) und die Quelle ordnungsgemäß nennen, einen Link zur Creative Commons Lizenz beifügen und angeben, ob Änderungen vorgenommen wurden.

Die in diesem Artikel enthaltenen Bilder und sonstiges Drittmaterial unterliegen ebenfalls der genannten Creative Commons Lizenz, sofern sich aus der Abbildungslegende nichts anderes ergibt. Sofern das betreffende Material nicht unter der genannten Creative Commons Lizenz steht und die betreffende Handlung nicht nach gesetzlichen Vorschriften erlaubt ist, ist für die oben aufgeführten Weiterverwendungen des Materials die Einwilligung des jeweiligen Rechteinhabers einzuholen.

Weitere Details zur Lizenz entnehmen Sie bitte der Lizenzinformation auf http://creativecommons.org/licenses/by/4.0/deed.de.

\section{Literatur}

Ackermann C, Beggiato M, Schubert S, Krems JF (2019) An experimental study to investigate design and assessment criteria: What is important for communication between pedestrians and automated vehicles? Appl Ergon 75:272-282

Bangerter A, Mayor E (2013) Interactional theories of communication. In: Cobley P, Schulz PJ (Hrsg) Theories and models of communication. De Gruyter, Berlin, Bosten, S 257-271

Bazilinskyy P, Dodou D, de Winter J (2019) Survey on eHMI concepts: the effect of text, color, and perspective. Transp Res Part F 67:175-194

Beggiato M, Witzlack C, Krems JF (2017) Gap acceptance and time-toarrival estimates for informal communication between pedestrians and vehicles. In: Proceedings of the 9th international conference on automotive user interfaces and interactive vehicular applications, S 50-57

Charisi V, Habibovic A, Andersson J, Li J, Evers V (2017) Children's Views on Identification and Intention Communication of Self-driving Vehicles. In: Proceedings of the 2017 conference on interaction design and children, S 399-404 
Clark HH (2006) Social actions, social commitments. In: Enfield NJ, Levinson SC (Hrsg) Roots of human sociality: culture, cognition, and human interaction. Berg Press, Oxford, S 126-150

de Clerq K, Dietrich A, Núnez Valasco JP, de Winter J, Happee R (2019) External human-machine interfaces on automated vehicles: effects on pedestrian crossing decisions. Hum Factors 61(8): 1353-1370

Cloutier MS, Lachapelle U, d'Amours-Oulett AA, Bergeron J, Lord S, Torres J (2017) "Outta my way!" Individual and environmental correlates of interactions between pedestrians and vehicles during street crossings. Accid Anal Prev 104:36-45

Cohen J (1988) Statistical power analysis for the behavioral sciences, 2. Aufl. Erlbaum, Hillsdale

Colley M, Walch M, Gugenheimer J, Rukzio E (2019) Including people with Impairements from the start: external communication of autonomous vehicles. In: Proceedings of the 11th international conference on automotive user interfaces and interactive vehicular applications: adjunct proceedings, S 307-314

Deb S, Strawderman LJ, Carruth DW (2019) Should I cross? Evaluating options for autonomous vehicle and pedestrian interaction. In: Proceedings of the Road Safety and Simulation Conference

Dey D, Terken J (2017) Pedestrian interaction with vehicles: role of explicit and implicit communication. In: Proceedings of the 9th international conference on automotive user interfaces and interactive vehicular applications, S 109-113

Dey D, Walker F, Martens MH, Terken J (2019) Gaze patterns in pedestrian interaction with vehicles: towards effective design of external human-machine interfaces for automated vehicles. In: Proceedings of the 11th international conference on automotive user interfaces and interactive vehicular applications: adjunct proceedings, S 369-378

Eisma AB, van Bergen S, ter Brake SM, Hensen MTT, Tempelaar WJ, de Winter JCF (2020) External human-machine interfaces: the effect of display location on crossing intentions and eye movements. Information 11(1):1-18

ERTRAC (2019) Connected and automated driving roadmap. https:// www.ertrac.org/uploads/documentsearch/id57/ERTRAC-CADRoadmap-2019.pdf. Zugegriffen: 9. Jan. 2020

Fagnant DJ, Kockelman K (2015) Preparing a nation for autonomous vehicles: opportunities, barriers and policy recommendations. Transp Res Part A 77:167-181

Färber B (2016) Communication and communication problems between autonomous vehicles and human drivers. In: Maurer M, Gerdes JC, Lenz B, Winner H (Hrsg) Autonomous driving: technical, legal and social aspects. Springer, Berlin, Heidelberg, S $125-144$

Habibovic A, Lundgren VM, Andersson J, Klingegard M, Lagström T, Sirkka A, Fagerlönn J, Edgren C, Fredriksson R, Krupenia S, Saluäär D, Larsson P (2018) Communicating intent of automated vehicles to pedestrians. Front Psychol 9(1336):1-17

Imbsweiler J, Ruesch M, Weinreuter H, León FP, Deml B (2018a) Cooperation behaviour of road users in t-intersections during deadlock situations. Transp Res Part F 58:665-677

Imbsweiler J, Stoll T, Ruesch M, Baumann M, Deml B (2018b) Insight into the cooperation processes for traffic scenarios: modelling with naturalistic decision making. Cogn Technol Work 20:621-635

Joisten P, Alexandi E, Drews R, Klassen L, Petersohn P, Pick A, Schwindt S, Abendroth B (2019) Displaying vehicle driving mode - effects on pedestrian behavior and perceived safety. In: Proceedings of the 2nd international conference on human systems engineering and design, S 250-256

Kauffmann N, Winkler F, Naujoks F, Vollrath M (2018) "What makes a cooperative driver?" identifying parameters of implicit and explicit forms of communication in a lane change scenario. Transp Res Part F 58:1031-1042

Lee YM, Madigan R, Garcia J, Tomlinson A, Solernou A, Romano R, Markkula G, Merat N, Uttley J (2019) Understanding the messa- ges conveyed by automated vehicles. In: Proceedings of the 11th International Conference on Automotive User Interfaces and Interactive Vehicular Applications, S 134-143

Liu Q, Emmermann B, Suen O, Grant B, Hercules J, Glaser E, Lathrop B (2017) Rightward attentional bias in windshield displays: implication towards external human machine interfaces for selfdriving cars. In: 2017 IEEE Conference on Cognitive and Computational Aspects of Situation Management

Löcken A, Golling C, Riener A (2019a) How should automated vehicles interact with pedestrians? A comparative analysis of interaction concepts in virtual reality. In: Proceedings of the 11th International Conference on Automotive User Interfaces and Interactive Vehicular Applications, S 262-274

Löcken A, Wintersberger P, Frison A-K, Riener A (2019b) Investigating user requirements for communication between automated vehicles and vulnerable road users. In: IEEE Intelligent Vehicles Symposium (IV), S 879-884

Madigan R, Nordhoff S, Fox C, Ezzati Amini R, Louw T, Wilbrink M, Schieben A, Merat N (2019) Understanding interactions between automated road transport systems and other road users: a video analysis. Transp Res Part F 66:196-213

Merat N, Louw T, Madigan R, Wilbrink M, Schieben A (2018) What externally presented information do VRUs require when interacting with fully Automated Road Transport Systems in shared space? Accid Anal Prev 118:244-252

Merten K (1977) Kommunikationsprozesse im Straßenverkehr. In: Bundesministerium für Verkehr (Hrsg) Symposium Unfallforschung und Verkehrssicherheit. Schriftreihe Unfall- und Sicherheitsforschung Straßenverkehr. Heft 14:115-126

Millard-Ball A (2018) Pedestrians, autonomous vehicles, and cities. J Plan Educ Res 38(1):6-12

Morgenstern O (1968) Spieltheorie: Ein neues Paradigma der Sozialwissenschaft. Z Nationalökon 28:145-164

Nuñez Velasco JP, Farah H, van Arem B, Hagenzieker MP (2019) Studying pedestrians' crossing behavior when interacting with automated vehicles using virtual reality. Transp Res Part F 66:1-14

Penmetsa P, Adanu EK, Wood D, Wang T, Jones SL (2019) Perceptions and expectations of autonomous vehicles-A snapshot of vulnerable road user opinion. Technol Forecast Soc Chang 143:9-13

Rasouli A, Tsotsos JK (2020) Autonomous vehicles that interact with pedestrians: a survey of theory and practice. IEEE Trans Intell Transp Syst 21(3):900-918. 10.1109/TITS.2019.2901817

Reschke J, Auburger M-T, Marichalar R, Neumann C (2019) Kommunikation zwischen automatisierten Fahrzeugen und Fußgängern. ATZ Automob Z 121:16-23

Rouchitsas A, Alm H (2019) External human-machine interfaces for autonomous vehicle-to-pedestrian communication: a review of empirical work. Front Psychol 10(2757):1-12

Schieben A, Wilbrink M, Kettwich C, Madigan R, Louw T, Merat N (2019) Designing the interaction of automated vehicles with other traffic participants: design considerations based on human needs and expectation. Cogn Technol Work 21:69-85

Stanciu SC, Eby DW, Molnar LJ, St. Louis RM, Zanier N, Kostyniuk LP (2018) Pedestrians/bicyclists and autonomous vehicles: how will they communicate? Transp Res Rec 5:1-9

Strohm O, Ulich E (1997) Unternehmen arbeitspsychologisch bewerten. Ein Mehrebenenansatz unter besonderer Berücksichtigung von Mensch, Technik und Organisation. In: Schriftreihe Mensch, Technik, Organisation, Band. 10. vdf Hochschulverlag, Zürich

Verma H, Pythoud G, Eden G, Lalanne D, Evéquoz F (2019) Pedestrians and visual signs of intent: towards expressive autonomous passenger shuttles. Proc ACM Interact Mob Wearable Ubiquitou Technol 3(3): 107

Werner A (2018) New colours for autonomous driving: an evaluation of chromaticities for the external lightning equipment of autonomous vehicles. Colour Turn 1:1-14

Wilde GJS (1976) Social Interaction Patterns in Driver Behavior: An Introductory Review. Hum Factors 18(5):477-492 
Wintersberger P, Löcken A, Frison A-K, Riener A (2019) Evaluierung von Benutzeranforderungen für die Kommunikation zwischen automatisierten Fahrzeugen und ungeschützten Verkehrsteilneh- mern. In: Riener A, Appel A, Dorner W, Huber T, Kolb JC, Wagner (Hrsg) Autonome Shuttlebusse im ÖPNV. Springer Vieweg, Berlin Heidelberg, S 115-132 